ISSN electrónico: $1885-5210$

DOI: https://doi.org/10.14201/rmc202016e247259

\title{
ZOONOSIS. LA CARA OCULTA DE LA PANDEMIA COVID-19
}

\section{Zoonosis. The Hidden Face of the COVID-19 Pandemic}

\author{
Elías F. RODRÍGUEZ FERRIª Luis A. CALVO SÁEZ ${ }^{b}$ \\ ${ }^{a}$ Catedrático Emérito de Sanidad Animal (Microbiología e Inmunología). Universidad de León (España). \\ bPresidente del Consejo General de Colegios de Veterinarios de España (España). \\ Correo electrónico: ef.rferri@unileon.es; calvoluisalberto@gmail.com
}

Fecha de recepción: 3 de septiembre de 2020

Fecha de aceptación: 14 de septiembre de 2020

Fecha de publicación: 29 de enero de 2021

\section{Resumen}

Las zoonosis son enfermedades que se transmiten de forma natural entre los animales y el hombre. Su interés es enorme y creciente, especialmente en los últimos años en los que han emergido como problemas de gravedad y gran difusión, como zoonosis emergentes. Las zoonosis representan, al menos, el $60 \%$ de las enfermedades infecciosas humanas y hasta el $75 \%$ de las enfermedades emergentes, por lo que su importancia relativa esta sobradamente justificada. Desde el punto de vista sanitario son causa de muerte, enfermedad, sufrimiento y secuelas y, desde el punto de vista económico, causan quebranto en las naciones o, en todo el mundo, cuando la extensión de la enfermedad es global. El artículo discute cuestiones relativas a la predicción, prevención y control de zoonosis y se fija de modo particular, por su actualidad, en la COVID-19, una pandemia que tiene la condición de zoonosis al admitirse su origen en murciélagos con la intervención probable de un hospedador intermediario. Es importante la unión de fuerzas entre la sanidad animal, la salud humana y la sanidad ambiental, en el contexto de Una sola Salud, para lograr el éxito en el control y erradicación de estas enfermedades.

Palabras clave: zoonosis; salto de la barrera de especie; pandemia; epidemia.

\section{Abstract}

Zoonoses are diseases that are transmitted naturally between animals and man. Their interest is enormous and growing, especially in recent years in which they have emerged as 
serious and widespread problems, such as emerging zoonoses. Zoonoses represent at least $60 \%$ of human infectious diseases and up to $75 \%$ of emerging diseases, so their relative importance is more than justified. From the health point of view they are the cause of death, disease, suffering and sequelae, and from the economic point of view, they cause damage in nations or throughout the world, when the spread of the disease is global. The article discusses issues related to the prediction, prevention and control of zoonoses and is particularly set, due to its timeliness, in COVID-19, a pandemic that has the status of zoonosis by admitting its origin in bats. The union of forces between animal health, human health and environmental health, in the context of One Health, is important to achieve success in the control and eradication of these diseases.

Keywords: zoonosis; jumping the species barrier; Jump of the species barrier; pandemic; epidemic.

\section{ZOONOSIS}

El término zoonosis fue utilizado por Rudolph Virchow en el "Manual de Patología y Terapia Especial" publicado en 1855. Virchow, considerado el "padre" de la Patología Moderna y de la Patología Celular, estudió la triquinosis y señaló que entre la Medicina Humana y la Veterinaria no deberían haber líneas divisorias ${ }^{1}$.

Zoonosis (del griego zoon, animal y osis, enfermedad) alude a una enfermedad animal o que padecen los animales, sin más connotaciones. Su significado, sin embargo, refiere «una enfermedad transmisible al ser humano», una "enfermedad común o compartida, entre los animales o el hombre». Tal sería, incluso, que la dirección del contagio desde la fuente de infección (animal al hombre o el hombre al animal) quedaría amparada por el término, aunque algunos autores y la propia OMS (Organización Mundial de la Salud) definiesen el camino con términos complicados como "zooantroponosis» o "antropozoonosis» indicadores de la dirección del contagio, desde los animales al hombre o desde el hombre a los animales, aunque el uso o la tradición han preferido el término más corto, zoonosis, dándole un significado universal que no se corresponde con su etimología. Zoonosis, pues, refiere, una enfermedad infecciosa transmisible desde los animales al hombre, y también lo contrario.

En el Primer Informe de la reunión del Grupo Mixto OMS/FAO de Expertos en Zoonosis ${ }^{2}$ celebrada en Ginebra en diciembre de 1950, se definen las zoonosis como "enfermedades que se transmiten de forma natural entre los animales vertebrados y el hombre». El informe incorporaba una lista de más de 80 enfermedades y afirmaba que se trata de «una de las ramas principales de la Salud Pública Veterinaria». El citado grupo de expertos se refiere a la Salud Pública Veterinaria como un concepto "nuevo» que «describe el moderno concepto de responsabilidad de la Medicina Veterinaria respecto de la Salud Pública».

\section{ENFERMEDADES EMERGENTES. ZOONOSIS EMERGENTES. ANTECEDENTES}

El concepto de emergencia, se corresponde con las antiguas plagas que asolaron la humanidad en todas las épocas. Los ejemplos son numerosos, como la Peste Antonina, que tuvo lugar entre el 165 y el 180 d. C, en el Imperio Romano, para la que se ha calculado una mortalidad de más de 5 millones de víctimas, o la Plaga Justiniana en 540-542 d. C, en Bizancio, para la que se calculan entre 25 y 50 millones de víctimas (la cuarta parte de la población mundial de entonces), relacionada con las ratas. Nuevas epidemias de peste bubónica o peste negra asolaron Asia y Europa en el siglo XIV diezmando la población (50-100 millones de muertes), repetida después en otras épocas, como en China, en el siglo 19, también con pérdidas de vidas millonarias. En el 
siglo XIX fue el cólera y el siglo XX se estrenó con la gripe «Española» (1918; entre 50 y 100 millones de muertos), repetida después como gripe Asiática y gripe de Hong Kong, hasta la emergencia de SIDA en 1981.

Las enfermedades emergentes, refiere enfermedades nuevas (o aparentemente nuevas), muy difusibles, graves, o que se describen en regiones nuevas o que surgen mediante algún tipo de transmisión nueva, como puede ser a través del consumo de alimentos. El término se comenzó a utilizar con profusión en la década de los años 90, aunque la primera vez que se utilizó la denominación fue con ocasión de un brote de piroplasmosis equina, que tuvo lugar en América, en $1962^{3}$. En 1971, David Sencer publicó una revisión distinguiendo el origen bacteriano, fúngico, vírico y parasitario ${ }^{4}$. En 1976, en Filadelfia, se produjo un brote de una enfermedad nueva, después denominada legionelosis, en el curso de una reunión de veteranos de guerra norteamericanos. En 1981, Richard Krause publicó 'The Restless Tide: The Persistent Challenge of the Microbial World"s y después apareció el SIDA, un prototipo de enfermedad emergente.

En los años 90 hubo un gran interés por las enfermedades emergentes y los riesgos derivados de nuevos brotes de enfermedades infecciosas. En 1992, el Instituto de Medicina de los EE.UU., publicó el informe de un Comité de Riesgos Microbianos para la Salud, editado por Lederberg, Shope y Oaks ${ }^{6}$ considerado una referencia, que se ocupó, especialmente, de los factores de emergencia. En 1994, el Centro Nacional para las Enfermedades Infecciosas (NCID), publicó un nuevo informe titulado 'Addressing Emerging Infectious Disease Threats $^{7}$ y se puede decir que su culminación fue la edición en 1995 de la revista Emerging Infectious Diseases, editada por el CDC. En 2003, el Instituto de Medicina publicó una actualización de su Informe anterior bajo el título 'Microbial Threats to Health. Emergence, Detection and Response's.
En $2001^{9}$, revisado después en $2005^{10}$, el trabajo de varios autores del Instituto Rooslin de Edimburgo, pusieron de manifiesto que el $60 \%$, aproximadamente, de los patógenos humanos, eran de procedencia animal, lo que suponía que ese porcentaje de las enfermedades infecciosas humanas eran zoonosis, y que además, el $75 \%$ de las enfermedades infecciosas emergentes humanas, eran también zoonosis, cifras que se han venido utilizando desde entonces, como referencia para poner de manifiesto la importancia relativa mayoritaria de las zoonosis en las patologías infecciosas humanas.

Por otra parte, desde los años noventa, con los informes del Comité de Riesgos Microbianos para la Salud, en los EE. UU. de 1992 y 2003, a los que se podrían añadir otros (NCID/CDC, 1994; Taylor et al., 2001) se venía trabajando en la descripción de los factores que propiciaban situaciones de emergencia. Se incluyeron factores dependientes de los microorganismos patógenos (principalmente cambios de origen genético mediante mutación, recombinación y reordenamiento, que originaban nuevas variantes, con propiedades nuevas, en especial en lo que la virulencia se refiere), factores dependientes del ambiente, del clima, la industria, tecnológicos, etc., y sobre todo influencias humanas (factores antropogénicos) la suma de los cuales $y$, principalmente su coincidencia en un denominado modelo de convergencia ${ }^{11}$, facilitaba la emergencia al producirse saltos de la barrera de especie desde los animales al hombre y su adaptación consiguiente que permitía, después, la transmisión interhumana, paso necesario para que las emergencias adquiriesen dimensión en forma de epidemias o pandemias. El salto de la barrera de especie o salto de especie o transmisión cruzada, se produce, en tres fases progresivas ${ }^{12}$ (Morse et al., 2012), como se observa en la figura 1.

Una lista, no exhaustiva, de factores de emergencia, incluye, como se ha dicho, factores genéticos y biológicos, que se refieren tanto 


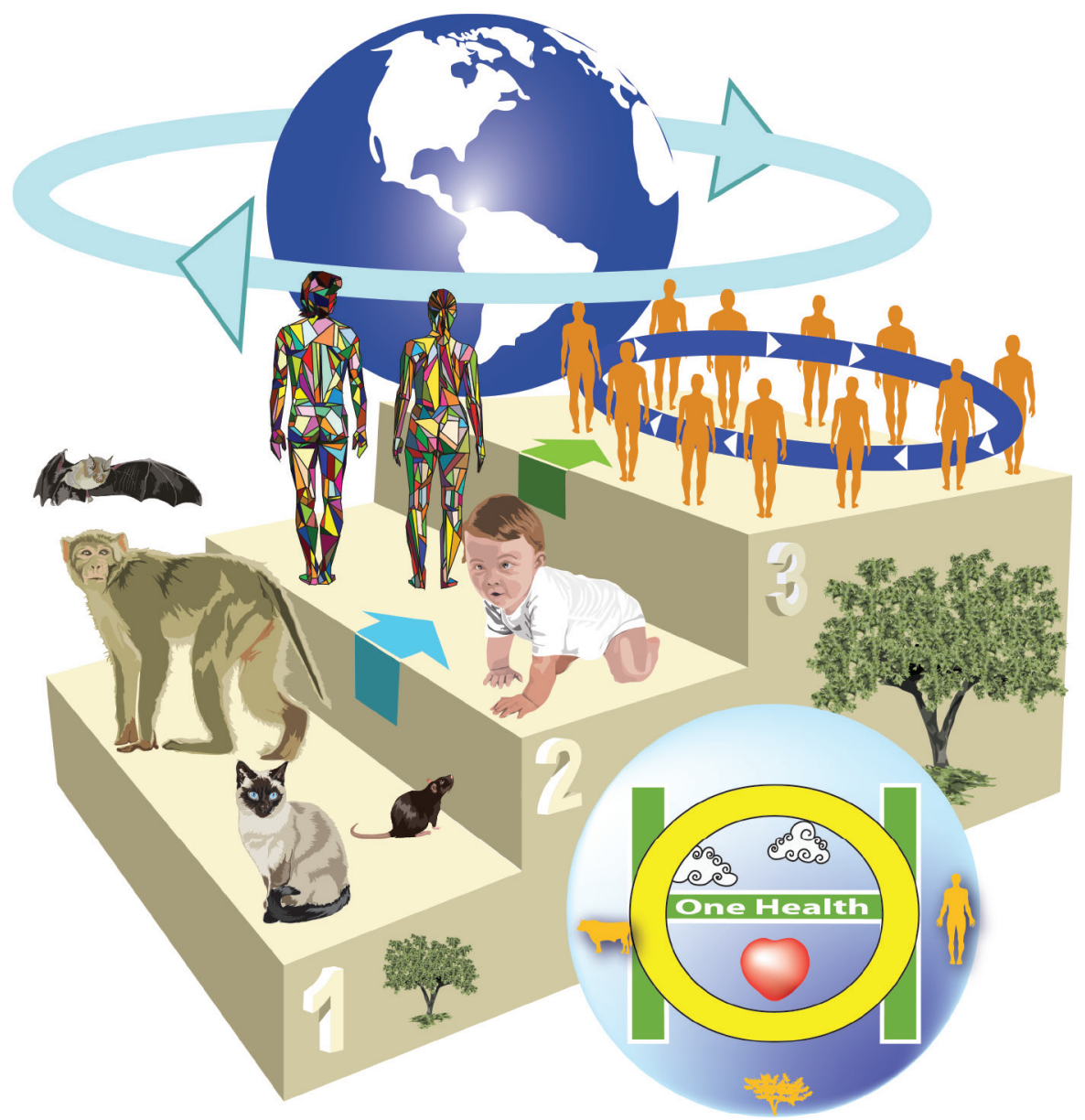

Figura 1. Las tres etapas del salto de la barrera de especie en las zoonosis, según Morse et al $(2012)^{27}$. En 1, el patógeno circula exclusivamente entre animales; en 2, se producen algunos saltos de barrera ocasionales al hombre, sin transmisión interhumana y en 3 , el patógeno se transmite entre los humanos, como consecuencia de su evolución en términos de adaptación al nuevo hospedador.

a los patógenos como a sus hospedadores; los primeros modifican bacterias, virus, etc., permitiéndoles evolucionar para adaptarse a las condiciones ambientales e incluyen el uso de mutaciones, recombinaciones $y$, en el caso de virus de genoma fragmentado, como los virus influenza, reordenamientos genéticos; respecto de los segundos, condicionan la susceptibilidad o resistencia, que permite o impiden la infección y su progresión hacia la enfermedad, como ocurre con los factores de la respuesta inmunitaria innata y la adaptativa o los receptores celulares, que permiten la infección y colonización celular. Entre los factores ambientales y físicos se incluye el clima y el tiempo climático, como fenómenos naturales, o los ambientes modificados por la 
mano del hombre, como ocurre en relación con el uso de la tierra, reforestación, deforestación, incendios forestales, construcciones (grandes embalses artificiales, carreteras, etc.) o simplemente, la incorporación del riego agrícola en tierras de secano. No menos importantes son los factores que se relacionan con la producción y transformación de alimentos, como la producción intensiva (en particular de animales salvajes o exóticos), la industria alimentaria o la tecnología de conservación o procesado. Existen, por último, factores sociales, políticos y económicos, que incluyen, por ejemplo, la pobreza y el hambre, las guerras, el comercio de animales (domésticos y salvajes), los viajes y el turismo, el poder y la desigualdad social, el daño intencionado (bioterrorismo), la falta de voluntad política o la falta de infraestructuras sanitarias.

Al tiempo, la emergencia de brotes y epidemias, abonaron iniciativas internacionales para la lucha, la prevención y el control de estos problemas que, en principio, se organizaron en torno a los brotes de gripe aviar producida por el virus influenza H5N1 (Programa de Prevención Global de la Influenza Aviar de Naciones Unidas, GPAI, cimentado sobre el previo de Coordinación de Naciones Unidas para la Influenza, UNSIC), que tuvieron lugar en el sudeste asiático desde 1997, recrudecidos en 2003, además de la emergencia de enfermedades producidas por virus nuevos como los virus Nipah y Hendra (del nuevo género Henipavirus), la encefalitis de West Nile (del Nilo Occidental), el SARS (síndrome respiratorio agudo grave) y los procesos por priones transmisibles (encefalopatía espongiforme bovina y la enfermedad debilitante del ciervo y el alce), propiciaron el nacimiento oficial de la nueva doctrina One Health (Una Salud o Una sola Salud) sobre los denominados Principios de Manhattan que proclaman una estrategia holística, integrada, de la lucha contra las zoonosis desde la perspectiva de la Salud Humana, la Sanidad Animal y la Sanidad Ambiental ${ }^{13}$. Precisamente ahora, con ocasión de la pandemia COVID-19 por el coronavirus SARSCoV-2, se reclama a los expertos para unir fuerzas frente a éste y otros problemas cuya magnitud sobrepasa las capacidades independientes de los países, igual que de las profesiones, implicadas en su lucha.

\section{SIGNIFICADO ACTUAL DE LAS ZOONOSIS}

EMERGENTES Y REEMERGENTES. IMPORTANCIA

SANITARIA, SOCIAL Y ECONÓMICA

Nadie puede dudar y mucho menos en los momentos que atravesamos, que las zoonosis emergentes representan un problema principal con connotaciones de tipo sanitario y económico, entre otras, que se complementan en las denominadas zoonosis reemergentes y las desatendidas. Las primeras son problemas controlados pero que ocasionalmente rebrotan y vuelven a saltar a primer plano de la actualidad por su importancia y, las segundas, se vinculan sobre todo con países subdesarrollados, tienen carácter endémico-crónico y no son objeto de medidas drásticas, en parte, por la falta de financiación, con el propósito de su eliminación, como sucede con la brucelosis, la rabia o algunas parasitosis.

Desde el punto de vista sanitario la principal anotación se refiere a la enfermedad y los fallecimientos derivados que, dada la dimensión del problema, se miden en decenas de miles o en millones, con las consecuencias correspondientes en términos de sufrimiento, dolor y secuelas, que al final se traducen también en discapacidades físicas y/o mentales que repercuten en su actividad y funcionalidad en términos de DALYS (Disability-Adjusted Life Years) años de vida ajustados por discapacidad.

Aún hoy, cumplido ya el primer quinto del nuevo siglo y milenio, las enfermedades infecciosas (y el $60 \%$ son zoonosis) siguen siendo responsables del $25 \%$ de los fallecimientos en el mundo (aproximadamente 10 millones de fallecimientos al año), donde los países subdesarrollados y particularmente tropicales son 
principales contribuidores ${ }^{14,15}$, mientras que en los países desarrollados se relegan a posiciones intermedias o secundarias. Según la OMS, mientras que en Europa la tasa de mortalidad infantil (menores de 5 años) fue del $9 \%$ o en 2018, en África el mismo valor alcanza la cifra del $76 \%$ o y en Oriente Medio del $47 \%$ o, unos valores que se atribuyen principalmente a responsabilidades infecciosas, ejemplo al que se pueden sumar los datos de las dos enfermedades que se consideran referencia en estos casos (Tuberculosis y Malaria), que en África alcanzan valores de 231 y 229,3 por cien mil habitantes respectivamente, mientras que en Europa tales cifras se sitúan en valores del 29 y 0 , respectivamente. Parece pertinente recordar, al respecto, que las regiones tropicales, entre el trópico de Cáncer y el de Capricornio, se concentra una ingente masa de población humana (hasta el $40 \%$ del total), con proyecciones al alza debido a una alta tasa de nacimientos y migraciones, en lo que coinciden condiciones (humedad y temperatura) que han favorecido tradicionalmente la emergencia de enfermedades infecciosas, una situación que puede ampliarse también a otras zonas próximas al trópico. Los ejemplos son numerosos, simplemente haciendo referencia a tres zonas concretas de África, Asia (sudeste asiático principalmente) y América Latina (SARS, Nilo Occidental, Chagas, Ébola, Dengue, Gripe Aviar, etc.), en su mayoría zoonosis $^{16}$, a los que se suman descripciones de patógenos nuevos como Rickettsia felis o Tropheryma whipplei, al lado de reemergencias de peste, cólera, Ébola, fiebre del Valle del Rift, etc. Otras zoonosis, como se señaló antes, endémicas o desatendidas, originan problemas persistentes sobre la salud humana a nivel regional en todo el mundo ${ }^{17}$, a la vez que son también endémicas en las poblaciones animales correspondientes, que sirven como fuente de infección para el hombre, aunque no se produzca la transmisión interhumana (o esta es rara) como sucede en la rabia o la tripanosomiasis, al contrario que otras, produciendo focos o brotes localizados o generalizados.

Las víctimas mortales de la gripe de 1918 se han estimado en más de 50 millones o la epidemia de SARS de 2002-03, que produjo 774 víctimas o el MERS surgido en 2012, que ya ha elevado la cifra de fallecimientos a 823 , o el brote de Ébola de 2013-16, que causó 11.310 fallecimientos en Liberia, Guinea y Sierra Leona y más de 2.000 en la República Democrática del Congo, - la pandemia de 2009 por el virus influenza H1N1, que produjo más de 18.000 víctimas, que han sido 32 millones en el caso de la pandemia de SIDA. Y todo eso, queda ya en segundo plano cuando vivimos la pandemia por COVID-19 que, en pleno progreso de rebrotes, ha elevado ya la cifra de contagios a casi 26 millones de casos y más de 850.000 fallecidos, sin poder saber, a fecha de hoy, cuál será su balance final.

En los últimos 70 años, la mayoría de las enfermedades emergentes identificadas han sido zoonosis, aunque su presencia haya sido escasa si se compara con las zoonosis endémicas, cuya distinción con las emergentes puede referirse tan solo a una cuestión temporal o geográfica. Así pues, una enfermedad (zoonosis) endémica, en una localización geográfica particular, puede ser considerada emergente

Las consecuencias económicas de las zoonosis no son de menor importancia y su impacto sobre la economía, puede ser muy elevado ${ }^{18}$. Simplemente una razón de número justificaría la afirmación pues, como se ha descrito, son excepcionales los vertebrados que no están implicados en una o más zoonosis y las pérdidas en vidas humanas o de animales, traducen siempre pérdidas económicas directas e indirectas; por ejemplo, en países desarrollados, muchas familias subsisten merced a los productos que obtienen de unos pocos animales (vacas, ovejas, cabras,...) y su muerte como consecuencia de enfermedad, además de suponer un riesgo de contagio por zoonosis, implica un 
desastre económico sin paliativos, que indirectamente también repercute en la salud humana. Se pierden recursos económicos en sectores de la producción agraria, en costes derivados de tratamientos, de prevención, costes derivados de la debilitación y pérdidas de productividad para el hombre, etc. Pero los costes van mucho más allá; por ejemplo, los quebrantos originados en el transporte, el comercio o el turismo y en la sociedad constituyen un capítulo importante del montante general que indirectamente repercuten en el bienestar y salud humanos.

Un estudio reciente del Servicio de Investigación del Parlamento Europeo ${ }^{19}$ se refiere a los costes de los sistemas de Salud Pública, públicos o privados, derivados del exceso ingresos hospitalarios, que, en el caso de la pandemia por H1N1 de 2009-11 supuso en UK, solo por este concepto, unos sobrecostes de más de 45 millones de libras, o las consecuencias de la crisis por EEB que ascendió a más de 3.400 millones de libras entre 1996 y 2000. El citado informe revisa otras epidemias, como el impacto de la fiebre del valle del Rift en Somalia, que produjo en 1997 una caída del PIB del 60-65\%, con una caída casi total de las exportaciones, que fueron evaluados en más de 300 millones de dólares, etc. Los ejemplos son, desde todos los puntos de vista, numerosos.

No hace mucho tiempo que algunos autores han estimado que los costes derivados de una pandemia grave, como la de gripe de 1918, podría alcanzar unos costes de más de 500.000 millones de dólares, por año, que representaría el $0,6 \%$ del ingreso bruto mundial ${ }^{20}$. También se han publicado otras estimaciones como, por ejemplo, que los costes derivados de la epidemia de SARS-CoV-1 pudieron haber supuesto cifras del orden de 30 a 50 mil millones de dólares, y eso que el número de afectados fue relativamente discreto ${ }^{21}$. En Liberia, como consecuencia del brote de Ébola entre 2013 y 2014, se consideró que el PIB (Producto Interior Bruto) pudo haber descendido desde el 8,7\% al 0,7\%, según estimaciones del Banco Mundial (https://www.worldbank.org /en/topic/macroeconomics/publication/2014 -2015-west-africa-ebola-crisis-impact-update) o en Sierra Leona del $5,3 \%$ hasta el $0,8 \%$, o en Guinea, del $4,1 \%$ al $0,1 \%$, unas cifras, como se ve, que representan el auténtico quebranto económico de estos países en subdesarrollo. Sin ir más lejos, el coste anual de las enfermedades transmitidas por alimentos (muchas de ellas zoonosis) se ha estimado entre 1,5 y $2,5 \mathrm{mil}$ millones de dólares, solo en USA y solo referido a 5 enfermedades principales, como salmonelosis (36\%) o campilobacteriosis (52\%). Esta última, la campilobacteriosis, producida por Campylobacter jejuni, la causa más importante en todo el mundo de gastroenteritis humana, que tiene en los animales (sobre todo en pollos de carne) su reservorio asintomático principal, mal conocida en clínica humana y con registros muy por debajo de los reales, podría representar, solo en la UE, con cifras proyectadas sobre reales ${ }^{22}$, más de 4.500 millones de euros, además de otros costes por secuelas que traducidos en unidades DALYs, supondría otros 2.400 millones adicionales.

Las primeras estimaciones sobre la COVID-19, no se han hecho esperar, aunque, por desgracia, todavía en plena pandemia, es difícil hacer un balance siquiera provisional y sus proyecciones, pero ya se hacen algunas declaraciones de personalidades internacionales, que estiman al alza una crisis económica sin precedentes.

Un portavoz del Banco Mundial, por ejemplo, en declaraciones realizadas el pasado 8 de junio, habla de parálisis económica, cuando los países impusieron las medidas de restricción de movimiento "se prevé una contracción del 5,2 por ciento del PIB mundial en 2020, utilizando ponderaciones del tipo de cambio de mercado, la recesión mundial más profunda en décadas. A más largo plazo, se espera que las profundas recesiones desencadenadas por la pandemia 
dejen cicatrices duraderas debido a una menor inversión, una erosión del capital humano debido a la pérdida de trabajo y educación y la fragmentación del comercio mundial y los vínculos de suministro" "se proyecta que las economías avanzadas se contraerán un $7 \%$, una debilidad que se extenderá a las perspectivas de las economías de mercados emergentes y en desarrollo, que se pronostica que se contraerán un $2,5 \%$ a medida que hagan frente a sus propios brotes nacionales del virus, lo que representaría el resultado más débil de este grupo de economías en al menos sesenta años» "Cada región está sujeta a degradaciones sustanciales del crecimiento: el este de Asia y el Pacífico crecerá un escaso 0,5\%, el sur de Asia se contraerá un 2,7\%, África subsahariana un 2,8 \%, Oriente Medio y norte de África un 4,2 \%, Europa y Asia central un 4,7\% y América Latina un 7,2 \%; recesiones que revertirán años de progreso y vuelvan a llevar a decenas de millones de personas a la pobreza extrema» «si persisten los brotes o si se prolongan las interrupciones de la actividad económica, la recesión podría ser todavía más profunda $»^{23}$. Otras opiniones, formuladas a finales de julio, no introducen mejores vaticinios. En un artículo publicado por McKinsey \& Company, que refiere resultados de una encuesta, se señala que, en América del Norte y en los mercados en desarrollo, las perspectivas se han continuado degradando desde el mes de junio y sugieren cautela e incertidumbre, con opiniones poco favorables sobre las perspectivas de recuperación ${ }^{24}$.

\section{LA COVID-19 ES UNA ZOONOSIS. EL PAPEL DE LOS ANIMALES SALVAJES}

El papel de los animales salvajes en el origen de las zoonosis, incluso en la transmisión de agentes patógenos a los domésticos, cuando se comparten algunos nichos ecológicos por razón de alimentación o bebida, dio un giro importante cuando se centró la atención en los mismos como causa de introducción de patógenos nuevos.
Daszak $^{25}$ (2000) en un artículo publicado en Science hizo referencia a lo que denominaba "polución de patógenos» para indicar el interés de los patógenos que vivían sin cambios clínicos en hospedadores salvajes que actuaban como reservorios para los animales domésticos y el hombre, interesándose por la necesidad de su control.

Las enfermedades infecciosas emergentes (EIE) de animales salvajes de vida libre pueden clasificarse en tres grupos sobre la base de criterios epizootiológicos clave: (i) EIE asociadas con saltos de barrera de animales domésticos a poblaciones salvajes que viven en el mismo entorno; (ii) EIE relacionadas directamente con la intervención humana, a través de traslocaciones de hospedadores o parásitos; y (iii) EIE sin participación manifiesta de humanos o animales domésticos. Estos fenómenos tienen dos implicaciones biológicas importantes: primero, muchas especies de vida silvestre son reservorios de patógenos que amenazan la salud humana y de los animales domésticos; en segundo lugar, las EIE originadas en la vida silvestre representan una amenaza sustancial para la conservación de la biodiversidad a nivel global.

La actual pandemia de COVID-19 tiene origen animal. Desde el principio se estableció su relación con los murciélagos, encontrándose que el SARS-CoV-2 mantenía un $96 \%$ de similaridad con un coronavirus de este origen (RaTG13-CoV), planteándose la posibilidad de un intermediario, también salvaje (pangolín o serpientes), que hubiera podido ser el eslabón necesario para su adaptación al hombre. En cualquier caso, todavía, ni del uno ni del otro, se han publicado pruebas definitivas. Por otra parte, a lo largo de la pandemia se han publicado algunos casos en animales de compañía, principalmente gatos y perros, contagiados por sus propietarios, igual que animales de zoo (tigre) o relacionados con la producción de pieles (visones), a los que se une la demostración de la susceptibilidad de hurones y hámsters, que ofrecen 
buenas perspectivas como animales modelo, para investigaciones de todo tipo.

\section{VIGILANCIA, PREVENCIÓN Y PREDICCIÓN DE ZOONOSIS PANDÉMICAS}

La vigilancia de zoonosis es la base de la prevención y ésta lo es del control, formando parte de la misma los desarrollos dirigidos a predecir su aparición cuando coinciden determinados factores de riesgo. Los recursos de laboratorio dependientes de la etiología, aplicados al aislamiento e identificación del agente, tanto como los métodos moleculares o genéticos, los métodos serológicos de base inmunitaria y las herramientas matemáticas base de los análisis eco-epidemiológicos, representan el proceder habitual. Así, por ejemplo, es importantes conocer cómo se alteran las poblaciones animales, en especial, la fauna salvaje y su microbiota, en particular virus o bacterias dotados de características genéticas que se puedan relacionar con riesgo de transmisión al hombre y su difusión. Se diseñan modelos, a partir de estos conocimientos, para el estudio de la transmisión cruzada desde la fauna salvaje, que reproduzcan el salto de la barrera de especie, que tracen el itinerario desde el origen animal al destino final humano la evolución de las zoonosis, analizando las causas y los factores determinantes y que sean capaces de evaluar su difusión (Woolhouse et al., 2005) ${ }^{26}$, pudiendo aproximarse previsiones sobre la emergencia de zoonosis.

Algunos grupos de investigadores, como el que lideran Peter Daszak y Stephen Morse ${ }^{27}$, de Ecohealth Alliance y la Escuela de Salud Pública de la Universidad de Columbia, en Nueva York, utilizan metaanálisis ${ }^{28}$ para el estudio de los resultados de detección y otros, que les ha permitido identificar puntos críticos pandémicos o de riesgo especial (hotspots), donde la coincidencia de factores, incluyendo socioeconómicos y ecológicos, puede facilitar la emergencia. Se pueden aplicar en programas vigilancia, aislando e identificando los potenciales patógenos, la secuenciación y comparación de su genoma con bancos de datos de agentes similares o próximos, etc. Se han identificado numerosos puntos críticos en áreas tropicales y subtropicales, con abundancia de fauna salvaje (Morse et al, 2014). El PREDICT ${ }^{29}$ es un programa de vigilancia de la Universidad de Davis, en California, que permiten cribar especies salvajes que se saben hospedan patógenos que han emergido en otras ocasiones, centrando después la atención en zonas calificadas como críticas, donde se produce el mayor número de exposiciones con el hombre, valorando condiciones que favorecen el salto de especie (proximidad filogenética con el hombre y otras), datos de la secuencia del genoma, antecedentes de otros patógenos con alguna relación, etc.

El Ilamado Global Preparedness Monitoring Board (GPMB) o Junta de Vigilancia Mundial para la Preparación (de zoonosis epidémicas y pandémicas) fue cofundada en 2018 por la OMS y el Banco Mundial, como un equipo especializado de alto nivel de "Respuesta Mundial a las Crisis Sanitarias» desde la de Ébola de 2014-16 y su objetivo principal es instar a la clase política para prepararse ante las emergencias sanitarias de ámbito mundial y mitigar sus efectos. Utiliza tres acciones principales: a) evaluación de la capacidad mundial para protegerse de las emergencias infecciosas; b) diagnosticar deficiencias críticas en la preparación frentes a las epidemias y c) promover con los políticos e instancias decisorias nacionales e internacionales, actividades de preparación. Está presidida por la ex Primera Ministra Noruega y Directora General de la OMS, la Dra. Gro Harlem Brundtland y Elhadj As Sy, Secretario General de la Cruz Roja.

El Primer Informe ${ }^{30}$, publicado en septiembre de 2019, contiene en su prólogo afirmaciones estremecedoras dirigidas a la clase política mundial, especialmente cuando se lee en el contexto de padecimiento de la actual pandemia de COVID19; por ejemplo: «la enfermedad se encuentra a 
sus anchas en el desorden y aprovecha la situación: los brotes de enfermedades han ido en aumento en las últimas décadas y el espectro de una emergencia sanitaria mundial se vislumbra peligrosamente en el horizonte», "nos enfrentamos a la amenaza muy real de una pandemia fulminante, sumamente mortífera, provocada por un patógeno respiratorio que podría matar de 50 a 80 millones de personas y liquidar casi el $5 \%$ de la economía munidal", "sería una catástrofe y desencadenaría caos, inestabilidad e inseguridad generalizadas, y el mundo, no está preparado».

Parece pues, que la profecía se ha cumplido, aunque no, afortunadamente en su virulencia y mortalidad, en los términos temidos. La enfermedad $X$ vaticinada por los expertos de la OMS $y$ de otros reconocidos expertos en $2015^{31}$ y mantenida en la revisión de $2018^{32}$, entre 7 enfermedades priorizadas, a la vista de la pandemia, no se puede afirmar, que se haya hecho mucho o suficiente, para hacer frente a las previsiones. Como se ha señalado al principio, en el comienzo de septiembre de 2020 llevamos ya casi 26 millones de casos de COVID-19 y una lista de fallecidos que supera los 850.000 y uno busca razones para una eficacia que no encuentra en los responsables políticos nacionales e internacionales en una situación previsible, diagnosticada por la Ciencia y la Gestión independientes, que fue apartada de las prioridades de forma inconsciente y cuyas consecuencias no resultan fáciles de imaginar, a todos los niveles.

\section{UNA SOLA SALUD}

El movimiento, iniciativa o doctrina conocido como Una Salud (One Health) proclama una estrategia holística, integrada, de la lucha contra las zoonosis desde la perspectiva de la Salud Humana, la Sanidad Animal y la Sanidad Ambiental. Como se ha señalado, Una sola Salud surgió como una necesidad para hacer frente a las zoonosis emergentes pandémicas. Como se recoge en un reciente editorial publicado en The Lancet $^{33}$, un equipo de expertos en fauna salvaje y veterinarios publicaron ${ }^{34}$ una extensa lista de procedimientos para promover el cambio de nuestras interrelaciones con los animales y para reducir el riesgo de otra pandemia similar a la que ahora sufrimos. Entre las opciones que se han considerado se ha hecho referencia a la necesidad de leyes para prevenir la mezcla de diferentes especies animales, la mejora de la sanidad animal en las explotaciones animales, limitando las densidades asegurando altos estándares de cuidados veterinarios y bienestar animal y cambio a una dieta más basada en plantas conviniendo que, incluso, una estrategia convencional podría ser insuficiente para abordar completamente las complejidades de las zoonosis. Para ayudar a los países a implementar una estrategia basada en Una Salud, se ha organizado una colaboración tripartita entre la OMS, la FAO y la OIE a la que la incorporación de un programa ambiental de Naciones Unidas podría fortalecer. Una verdadera estrategia de Una Salud, como se ha podido comprobar en el caso de enfermedades concretas, supondría desarrollar acciones conjuntas en actividades científicas y sociales, antropológicas, económicas y otras, sobre vigilancia, capacidad de construcción y reducción de riesgos, con participación de representantes de la industria, los viajes o el turismo, entre otros, pongamos por caso. Coincidimos con quienes piensan que esta pandemia es una advertencia frente a la explotación, sin medida, de la naturaleza y las zoonosis afectan, no solo a la salud, sino también a todo el tejido de la Sociedad. Nos tememos que COVID-19 no sea ni la última ni la peor pandemia zoonósica. El Cambio Climático, que se ha mostrado ya como una amenaza existencial puede galvanizar una respuesta de urgencia. Las zoonosis también necesitan algo así.

\section{REFERENCIAS}

1. Cordero del Campillo M. Comentarios sobre la Historia de las Zoonosis. En Álvarez M y Rodríguez Ferri E, Directores. Zoonosis. II Curso sobre Enfermedades 


\section{ZOONOSIS. LA CARA OCULTA DE LA PANDEMIA COVID-19 \\ ELÍAS F. RODRÍGUEZ FERRI; LUIS A. CALVO SÁEZ}

Transmisibles entre los Animales y el Hombre. Leon: Servicio de Publicaciones. Universidad de León; 2002. p. 9-28.

2. Organización Mundial de la Salud. Grupo Mixto OMS/FAO de Expertos en las Zoonosis. Informe de la Primera Reunión. Serie de Informes Técnicos número 40.1951.

3. Maurer FD. Equine piroplasmosis--another emerging disease. J Am Vet Med Assoc. 1962; 141:699-702.

4. Sencer DJ. Emerging diseases of man and animals. Annu Rev Microbiol 1971; 25: 465-86.

5. Krause RM. The restless tide: The persistent challenge of the microbial world. National Foundation for Infectious diseases. 1981. Library of Congress No.82-116089.

6. Lederberg J, Shope RE, Oaks SC (Edit). Emerging Infections. Microbial Threats to Health in the United States. Washington (DC): National Academies Press (US); 1992.

7. NCID/CDC. Addressing Emerging Infectious Disease Threats. A prevention strategy for the United States. 1994.

8. Smolinky MS, Hamburg MA Lederberg J. (Edit). Microbial Threats to Health. Emergence, Detection and Response. Institute of Medicine (US). Committee on Emerging Microbial Threats to Health in the $21^{\text {st }}$ Century. Washington (DC): National Academic Press (US) ; 2003.

9. Taylor LH, Latham SM, Woolhouse MEJ. Risk factor for human disease emergence. Phil Trans R Soc London B. $2001 ; 356$ (1411): 983-9.

10. Woolhouse MEJ, Gowtage-Sequeria S. Host range and emerging and reemerging pathogens. Emerg Infect Dis. $2005 ; 11(12): 1842-46$.

11. King LJ. Un modelo de convergencia para la aparición de enfermedades emergentes y reemergentes. 72a Sesión OIE. Paris 23-28 de mayo de 2004.

12. Wolfe ND, Dunavan CP, Diamond J. Origins of major human infectious diseases. Nature 2007; 447 (7142): 279-83.

13. Rodriguez Ferri EF. “Una Salud”. La colaboración es necesaria. Anales de la Real Academia de Doctores de España. 2013; 27(1), 205-26.
14. World Health Statistics 2020. Monitoring Health for the SDGs. Sustainable Development Goals. Geneva: WHO; 2020.

15. Dye C. After 2015 infection diseases in a new era of health and development. Phil Trans R Soc Lond B Biol Sci 2014;369(1645):20130426.

16. Torres JR. Emergence of Infectious Diseases in the 21th Century. Medicine and Health. 2012; Chapter IV. p. 119-21.

17. International Livestock Research Institute. Mapping of poverty and likely zoonoses hotspots. Zoonoses Project 4. Department for International Development, UK. Nairobi, Kenya: Int Livestock Res Inst; 2012.

18. Jones KE, Patel NG, Levy MA, Storeygard A, Balk D, Gittleman JL et al.Global Trends in emerging infection diseases. Nature 2008; 451 (7181): 990-3.

19. Delivorias A, Scholz N. Economic Impact of Epidemics and Pandemics. European Parliamentary Research Service PE 646.195-Feb 2020.

20. Fan VY, Jamison DT, Summers LH. Pandemic risk: how large are the expected losses?. Bull World Health Organ. 2018; 96(2): 129-34.

21. Newcomb J, Harrington T, Aldrich S. The economic impact of selected infectious disease outbreaks. Cambridge MA: Bio Economic Res Associated; 2011.

22. Buzby C, Roberts T. Estimated annual costs of Campylobacter Associated Guillain-Barré Syndrome. Agricultural Economic Report 756. Washigton DC: USDA/ERS; 1997

23. World Bank. The Global Economic Outlook During the COVID-19 Pandemic: A Changed World. World Bank Group [Internet] June, 8. 2020.

24. McKinsey and Company. The coronavirus effect on global economic sentiment. July 27, 2020.

25. Daszak P. Cunningham AA, Hyatt AD. Emerging infectious diseases of wildlife -threats to biodiversity and human health. Science. 2000; 287 (5452). 443-49.

26. Davis S, Begon M, De Bruyn L, Ageyev VS, Klassovskiy NL, Pole SB et al. Predictive thresholds for plague in Kazakhstan. Science. 2004; 304(5671): 736-8. 


\section{ZOONOSIS. LA CARA OCULTA DE LA PANDEMIA COVID-19 \\ ELÍAS F. RODRÍGUEZ FERRI; LUIS A. CALVO SÁEZ}

27. Morse SS, Mazet JAK, Woolhouse M, Parrish CR, Carroll D, Karesh WB, Zambrana-Torrelio C et al. Prediction and prevention of the next pandemic zoonosis. Lancet. 2012; 380: 1956-65.

28. Marín Martínez F, Sánchez Meca J, López López JA. El metaanálisis en el ámbito de las ciencias de la Salud: una metodología imprescindible para la eficiente acumulación del conocimiento. Fisioterapia.2009; 31(3):107-14.

29. PREDICT: building a global early warning system for emerging diseases that move between wildlife and people. September 27, 2012.

30. GPMB (Global Preparedness Monitoring Board). Un mundo en peligro. Informe anual sobre preparación mundial para las emergencias sanitarias. Junta de
Vigilancia Mundial de la Preparación. Geneva: WHO; 2020;2019.

31. WHO. Blueprint for R\&D preparedness and response to public health emergencies due to highly infectious pathogens Workshop on Prioritization of Pathogens. WHO [Internet] 15 December 2015.

32. WHO Research and Development Blueprint. 2018 Annual review of diseases prioritized under the Research and Development Blueprint Informal consultation 6-7 February 2018 Geneva, Switzerland

33. The Lancet. Zoonoses: beyond the human-animal-environment interface. 2020; 396: 1.

34. Petrovan SO, Aldridge DC, Bartlett $H$, Bladon A, Booth H, Broad S, et al. "Post COVID-19: a solution scan of options for preventing future zoonotic epidemics". OSF; 2020. 


\begin{tabular}{|l|l|} 
Elías F. Rodríguez Ferri. Licenciado y Dr. en Veterinaria por la Universidad \\
de Oviedo (León), catedrático de Microbiología e Inmunología en la Uni- \\
versidad Complutense (Veterinaria) y de Sanidad Animal (Microbiología \\
e Inmunología) en la Universidad de León (Veterinaria). Secretario de la \\
Facultad de Veterinaria de Madrid y Decano en la de León, Director del \\
Departamento de Sanidad Animal y Director Gerente del Instituto de Bio- \\
tecnología. Director de 19 Tesis Doctorales con importantes Premios y autor \\
de más de 300 artículos (investigación, doctrinales, sanitarios, divulgación, \\
etc.) y varios libros y capítulos de libros. Investigador Principal de más de \\
40 proyectos competitivos y contratos. Miembro del Consejo Asesor de la \\
Agencia Española del Medicamento, del Comité Científico de la Agencia \\
Española de Seguridad Alimentaria y del Ministerio de Ciencia y Tecnolo- \\
gía (Profarma II) y de la Consejería de Agricultura y Ganadería de Castilla \\
y León. En la actualidad es Catedrático Emérito. Pertenece a las Reales \\
Academias de Doctores y Ciencias Veterinarias de España, de la Real Aca- \\
demia Nacional de Medicina y la Real Academia Sevillana de Veterinaria. Es \\
Presidente y Fundador de la Academia de Ciencias Veterinarias de Castilla \\
y León. Ha recibido otros premios y distinciones.
\end{tabular}

